\title{
gynécologie
}

Société Suisse de Gynécologie et d'Obstétrique

Schweizerische Gesellschaft für Gynäkologie und Geburtshilfe

Società Svizzera di Ginecologia e Ostetricia

http://www.sggg.ch

Präsidentin

Dr. med. Franziska Maurer-Marti

Frauenklinik

Bürgerspital Solothurn

Schöngrünstrasse 42

CH-4500 Solothurn (Schweiz)

Tel. +41 3262744 71, Fax +41326274473

E-Mailfmaurer_so@spital.ktso.ch
Generalsekretärin

Dr. med. Christiane Roth

Im Obstgarten 11

CH-8044 Gockhausen (Schweiz)

Tel. +41433338411

E-Mailinfo@christiane-roth.ch

\section{Liebe Kolleginnen und Kollegen}

\section{Liebe Freunde}

Im Dezember 2007 wurde bei den Mitgliedern von gynécologie suisse eine Umfrage durchgeführt.

Das Ziel des Vorstandes war damals, die Meinung von möglichst vielen Kollegen und Kolleginnen kennenzulernen und die Akzeptanz gewisser - angedachter - Zukunftsstrategien zu erfragen.

Als Ergebnis zeigte sich neben dem Wunsch nach Änderung in der gesellschaftsinternen und externen Kommunikation auch die Befürwortung der Professionalisierung des Generalsekretariats.

Anlässlich der Generalversammlung im Juni 2008 hat die Wahl von Frau Dr. Christiane Roth stattgefunden.

Gerne möchte ich Ihnen unsere Generalsekretärin, welche ihre neue Aufgabe mit grossem Engagement aufgenommen hat, persönlich vorstellen:

Christiane Roth war viele Jahre in leitenden Funktionen im Spitalmanagement tätig. Von 2000 bis 2008 als Spitaldirektorin des Universitätsspitals Zürich, von 1995 bis 2000 hat sie als Direktionspräsidentin die Universitären Psychiatrischen Dienste in Bern geleitet, davor war sie als Adjunktin des ärztlichen Direktors, Prof. Hans Bossart, des Universitätsspitals Lausanne tätig. Aus dieser Zeit und aus der Tätigkeit als Leiterin der Abteilung Weiter- und Fortbildung der FMH hatte sie gute und intensive Kontakte zur SGGG, die sie als innovative, fortschrittliche Fachgesellschaft in bester Erinnerung hatte. Christiane Roth ist ausgebildete Ärztin und im Besitz von Facharzttiteln in Pathologie und klinischer Zytologie. Berufsbegleitend hat sie von 1992 bis 1994 das Nachdiplomstudium «Management im Gesundheitswesen» absolviert. Im Rahmen ihrer ärztlichen Tätigkeit, sowie auch durch ihre Führungsaufgaben hat sie sich umfassende Kenntnisse im Schweizer Gesundheitswesen erworben. Seit März 2008 ist sie selbständig in der eigenen Firma tätig und übernimmt Projekte und Beratungsmandate im Gesundheitswesen und verwandten Gebieten. Christiane Roth ist Mutter von drei erwachsenen Kindern, wohnt in Gockhausen und ist Mitglied eines Rotaryclubs in Zürich. Neben Kunst und Kultur interessiert sie sich für Blumen und Gärten, reist gerne, bekocht mit Liebe ihre Gäste und liest in ihrer Freizeit am liebsten Biographien.

Die Generalsekretärin ist über info@christiane-roth.ch und/ oder über Tel. 0433338411 mit Combox erreichbar, damit Sie zurückgerufen werden können, wenn das Telefon nicht abgenommen werden kann.

In den letzten 6 Monaten hat uns Folgendes hauptsächlich beschäftigt:

\section{Kommunikation}

Wie erwähnt soll die Kommunikation der SGGG intern und nach aussen möglichst rasch verbessert werden. Um einen möglichst professionellen Auftritt zu erreichen, hat der Vorstand die Agentur Forum PR in Bern beauftragt, ein Konzept zu entwickeln. Das Forum PR arbeitet unter anderem auch für die Zahnärzte (SSO) und die Ärztegesellschaft des Kantons Bern, hat also Erfahrung mit Institutionen des Gesundheitswesens.

Zusammen mit Herrn F. Adank, eidg. diplomierter PR-Berater, haben sich die Mitglieder des Vorstandes anlässlich eines Strategieworkshops intensiv mit der Problematik auseinandergesetzt.

Im Workshop wollte Herr Adank die Vorstandsmitglieder und die Anliegen der SGGG kennenlernen. In kleinen Arbeitsgruppen fragte er die Werte/Wertvorstellungen der SGGG ab und im Anschluss daran stellte er 5 Fragen, die in Zweiergruppen beantwortet und im Plenum präsentiert und diskutiert wurden. So

\section{KARGER}

(C) 2009 S. Karger AG, Basel

Fax +41613061234 
wollte er wissen, welches die bisherigen Vorteile einer Mitgliedschaft bei der SGGG sind, welches zu schaffende Anreize für eine Mitgliedschaft sind, welche Elemente in ein Leitbild gehören müssten, welche Kommunikationsziele erreicht werden sollen und welche Zielgruppen zu definieren sind. Mit den gruppenweise präsentierten Anworten hat Herr Adank die notwendigen Inputs erhalten, die es ihm ermöglichen, das Grobkonzept für die Kommunikation zu entwickeln.

Ein wichtiger Teil in der Kommunikation ist die Homepage, die ebenfalls überarbeitet und neu strukturiert wird. Ziel ist es ein Instrument zu schaffen, welches den Mitgliedern der SGGG es einerseits ermöglicht, rasch zu den für sie wichtigen Informationen zu gelangen, und mit dem sie andererseits so viel wie möglich auf elektronischem Weg ohne Abhängigkeit von Bürozeiten und telefonischen Auskünften erledigen können.

Ein Schwerpunkt der neuen Homepage soll auch das Auftreten nach aussen sein.

Die Generalsekretärin wird in Zukunft die Koordination für die Homepage übernehmen und in Zusammenarbeit mit den Mitgliedern Texte bearbeiten, aufschalten etc. Wir werden Sie fortlaufend informieren und Ihnen (spätestens) anlässlich der Jahresversammlung in Lugano das Kommunikationskonzept und die Homepage präsentieren.

\section{Diagnosebasierte Fallpauschalen}

Im Rahmen der neuen Spitalfinanzierung hat der Bundesrat entschieden, ab 2012 die stationären Aufenthalte in den Akutspitälern mittels Fallpauschalen zu entschädigen. In Anlehnung an Deutschland und basierend auf den bisher gemachten Erfahrungen werden Gruppen von Krankheiten und Krankheitsbildern gebildet (DRG, «diagnosis-related group») und mit einem Preis versehen. Dieses System wird SwissDRG genannt.

D. Musfeld, Mitglied des Vorstandes, ist verantwortlich für DRG und hat darüber informiert, dass am 15. Oktober letzter Eingabetermin für das Einreichen der Anträge zu den CHOP-Erweiterungen (Schweizerischer Prozedurenkatalog) bei der FMH ist.

Da der deutsche OPS-Katalog (Operationen- und Prozedurenschlüssel) im Vergleich zum schweizerischen sehr viel mehr Prozeduren ausweist, mussten in einem ersten Schritt die FMCHFachgesellschaften die in der Schweiz relevanten Prozeduren im deutschen OPS-Katalog kennzeichnen. Zusammen mit einem Oberarzt der Frauenklinik am Universitätsspital Basel, welches als Referenzspital dient, hat D. Musfeld die Listen durchgesehen und an Dr. Bartkowski, deutscher Experte für DRG-Fragestellungen, der von der FMCH als Teilzeitmitarbeiter verpflichtet werden konnte, zugestellt.

In einem zweiten Schritt hat Dr. Rolf Bartkowski diese Leistungen in DRG-relevante Gruppen zusammengefasst, die in der Regel zu beantragenden neuen CHOP-Codes entsprechen. Die FMH wird sodann die Listen an das Casemix Office zustellen, wo die Anträge weiter bearbeitet werden.

Der Vorstand und der Delegierte für DRG werden aufmerksam verfolgen, wie es weitergeht und Sie auf dem Laufenden halten. Für uns ist wichtig, dass in der Gynäkologie und in der Geburtshilfe die Aktivitäten umfassend abgebildet sind und vor allem, dass zukünftige, innovative Behandlungsmöglichkeiten frühzeitig in das System eingespeist werden. D. Musfeld hat viel Arbeit geleistet, die herzlich verdankt wird.

Neues Bundesgesetz über Prävention und Gesundheits-

förderung und neues Bundesgesetz über das Schweizerische Institut für Prävention und Gesundheitsförderung

Der Bundesrat hat am 25.Juni 2008 das Eidgenössische Departement des Innern beauftragt, bei den Kantonen, den politischen Parteien, den gesamtschweizerischen Dachverbänden der
Gemeinden, Städte und Berggebiete, den Dachverbänden der Wirtschaft und den interessierten Kreisen ein Vernehmlassungsverfahren zu einem neuen Bundesgesetz über Prävention und Gesundheitsförderung und einem neuen Bundesgesetz über das Schweizerische Institut für Prävention und Gesundheitsförderung durchzuführen.

Nach dem Scheitern des ersten Vorschlags im Jahr 1984 galt das Augenmerk der Gesundheitspolitik vornehmlich der kurativen Medizin und der Finanzierung der Versorgungssysteme. Aufgrund der aktuellen gesundheitspolitischen Herausforderungen, die sich aus der demografischen Entwicklung, der Zunahme chronischer Krankheiten und der Wiederkehr bzw. dem Neuauftreten von Infektionskrankheiten ergeben, sind seit einiger Zeit auf nationaler Ebene Bestrebungen zu erkennen, die Prävention und die Gesundheitsförderung zu stärken.

Dazu haben wir im Rahmen des Vernehmlassungsverfahrens Stellung genommen. Gerne fügen wir diese hier an:

Der Vorstand von gynécologie suisse begrüsst ausdrücklich die Schaffung des Präventionsgesetzes und die Regelung der enthaltenen Kernelemente. Damit wird es möglich, Prävention und Gesundheitsförderung als eigenen, umfassenden Wirkungskreis zu bestätigen und diesen der Krankenversorgung gegenüberzustellen.

Wichtig scheint uns, dass über das Gesetz die Koordination und Kooperation zwischen Bund, Kantonen und allen anderen öffentlichen und privaten Akteuren sichergestellt und in methodischen und fachlichen Fragen Unterstützung bereitgestellt wird. Heute gibt es bereits viele Aktivitäten, die von Seiten der Leistungserbringer im Sinne der Prävention erbracht, aber nicht direkt als solche wahrgenommen werden (Beratungsgespräche beim Kinder- oder Frauenarzt als Beispiele). Mit dem Gesetz wird es möglich sein, Lücken zu schliessen im Bereich der nicht übertragbaren und psychischen Krankheiten und sowohl für das Individuum als auch für Gruppen mittels künftiger, geeigneter Massnahmen effizienter Wirkung zu entfalten.

Unser Anliegen ist es, dass der Entwicklung der Medizin und deren Auswirkungen auf das Individuum und die Gesellschaft - insbesondere wenn es um Früherkennung von Krankheiten geht - Rechnung getragen wird, aber auch ethische Fragestellungen benannt, ausdiskutiert und in die Entscheidfindung für zukünftige Massnahmen miteinbezogen werden. Als Ärztinnen und Ärzte, die sich mit der Gesundheit von Mutter und Kind befassen, liegt uns daran, dass nebst der Krebsvorsorge in der Gynäkologie auch der Schwangerenvorsorge inklusive Ultraschalluntersuchungen Genüge getan wird und dies nicht nur am Rande im Rahmen des Arbeitsplatzschutzes erwähnt wird.

Dies umso mehr, als Prof. Thomas Zeltner, Direktor des BAG, anlässlich des Ultraschallkongresses dieses Gesetz ausdrücklich auch im Zusammenhang mit der Schwangerschaft angekündigt hat. Ferner erinnern wir daran, dass wir im Zusammenhang mit Ultraschall seit Jahren ein Fehlbildungsregister ausgewählter Fehlbildungen verlangt haben; dies ist im Gesetz vorgesehen (Art. 20 und 21).

Wir begrüssen auch das Bundesgesetz über das Schweizerische Institut für Prävention und Gesundheitsförderung. Die Tatsache, dass eine öffentlich-rechtliche Anstalt im Besitz des Bundes (Institution des 3. Kreises) zum zentralen Ansprechpartner wird für die Kantone und private Präventions- und Gesundheitsförderungsorganisationen, bietet Gewähr, dass Kooperation und Koordination keine leeren Worthülsen bilden. Voraussetzung dafür ist, dass die Stiftung für Gesundheitsförderung und die Fachstelle zur Verwaltung der Tabakpräventionsabgabe ohne Wenn und Aber in das Institut integriert werden. Ferner müssen Aufgaben, Kompetenzen und Verantwortlichkeiten für das Insti- 
tut so definiert werden, dass kompetente Persönlichkeiten für die Direktion gefunden werden können. Die Schnittstellen mit anderen Institutionen müssen so definiert werden, dass kein unnötiges Konfliktpotential generiert wird.

Bezüglich der Kosten wird zugesichert, dass diese wegen der Aufgabenumlagerung bis 2012 nicht erhöht werden (müssen). Dies nehmen wir mit einer gewissen Skepsis zur Kenntnis. Sicher müssen der Finanzierung von Prävention und Gesundheitsförderung die notwendige Aufmerksamkeit und das nötige Gewicht zugemessen werden; dennoch ist es aus heutiger Sicht undenkbar, dass der Betrag für die Krankheitsverhütung (KVG) pro versicherte Person und pro Jahr von heute CHF 2.40 beliebig erhöht werden kann.

Als Präsidentin werde ich Sie gerne über weitere Aktivitäten Ihres Vorstandes informieren.

Für 2009 wünsche ich Ihnen beste Gesundheit und alles Gute.

Dr. Franziska Maurer-Marti

Präsidentin gynécologie suisse/SGGG 